\title{
Review \\ Overdiagnosis and overtreatment of breast cancer Microsimulation modelling estimates based on observed screen and clinical data
}

\author{
Harry J de Koning, Gerrit Draisma, Jacques Fracheboud and Arry de Bruijn
}

Erasmus MC, Department of Public Health, PO Box 1738, 3000 DR Rotterdam, The Netherlands

Corresponding author: HJ de Koning, h.dekoning@erasmusmc.nl

Published: 21 December 2005

This article is online at http://breast-cancer-research.com/content/8/1/202

(c) 2005 BioMed Central Ltd
Breast Cancer Research 2006, 8:202 (doi:10.1186/bcr1369)

of clinically relevant diseases; however, women may not necessarily benefit because they more often die due to other diseases.

This paper presents quantitative estimates of overdiagnosis in breast cancer screening based on microsimulation modelling, with special emphasis on DCIS. In this study, overdiagnosis is defined as diagnosing cancers that would not have been diagnosed clinically if there were no screening programme.

\section{Observations}

Starting to screen a population systematically for breast cancer will lead to the detection of cancers about three to four years earlier than without such an approach [6]; therefore, the number of detected cancers at the population level is expected to increase. Because screening is continued every consecutive year, this number is higher, and remains so, than if there were no systematic screening. Figure 1 shows the Dutch national data since 1989, when screening was gradually being implemented [7,8]. For women aged 50 to 69 years, implementation took place in the period 1990 to 1997. After an initial increase of around $30 \%$, incidence in the 50 to 69 year old age group stabilized at $16 \%$ higher than without screening. Furthermore, the last years of screening have resulted in an additional 10\% increase, probably due to more referrals and better screening performance. From 1999 onwards in the Netherlands women aged 70 to 74 were also invited for screening. Compared to the year 1989, the number of breast cancers diagnosed each year has increased by $40 \%$. Proportionally, this increase is largest for DCIS. Figure $2 a, b$ shows the increase in Dutch hospital admissions for non-invasive breast cancer in the years 1990 to 1992 (at the start of nation-wide screening) in municipalities that had started screening compared to municipalities that had not; in the age group invited for screening (at that time 50 to 69 year olds), the increase was

$\mathrm{DCIS}=$ ductal carcinoma in situ 


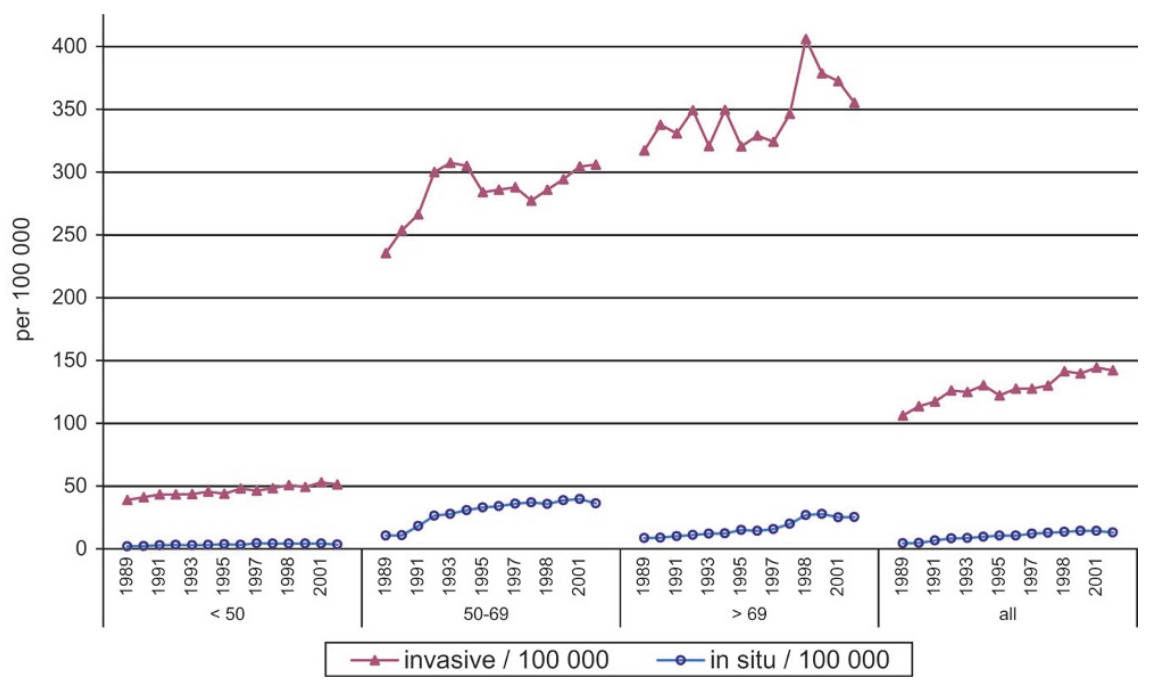

Age-specific incidence of breast cancer (invasive/non-invasive) from 1989 to 2002 in the Netherlands $[7,8]$.

3 to 5-fold (Fig. 2a). Strikingly, there was also an increase outside the screening municipalities (Fig. 2b). Non-invasive breast cancer, however, still accounted for only $4 \%$ of the total incidence [8].

\section{Modelling}

These increases in incidence represent real overdiagnosis to only a limited extent. From the observed rates, one can not easily determine to what extent overdiagnosis is involved because screening is still being continued. In these circumstances, modelling of the natural history of breast cancer and its early lesions, and what screening is estimated to depict, is crucial and provides a 'best guess'. Using the microsimulation model MISCAN [6,9], we first simulate individual life histories for women in the absence of screening and then assess how these histories would change as a consequence of a screening programme. The natural history is modelled as a progression from no breast cancer through pre-clinical disease (DCIS, T1a, 1b, 1c, T2+) to clinical disease (same stages). From a given pre-clinical state, a cancer may be detected by screening or become clinically apparent or, if undiagnosed, progress to the next pre-clinical state. To correctly model this natural history of breast cancer for women in a certain age group, one has to estimate mean durations of the different pre-clinical phases, transition probabilities, and sensitivity of the applied test [10]. Basically, one therefore needs data from two sources: observed screen and clinical data. These data include clinical incidence data by age and stage in the situation without screening, data on screen-detected cancers by stage, screening round (and interval) and age, and corresponding clinical incidence data when screening is being implemented [11]. Although the observed data can often be explained by a small range of parameters (e.g., a somewhat higher sensitivity and shorter mean duration of the stage may also result in a good fit), by having more detailed data from several screening rounds, by screening different age groups and/or by using different screening intervals, best parameters often fall into a smaller range [12]. In the Netherlands, such detailed data have been used: in the past using pilot data [6], and more recent data from the annual monitoring by the National Evaluation Team for Breast cancer screening [7].

The fit of the model to the breast cancer screening pilot data $[6,12]$, as well as to the Dutch nation-wide data [9], has been reported as quite satisfactory.

We also used the MISCAN approach to analyse the results of the Health Insurance Plan trial study. These comparisons show the potential power of modelling: the parameter values for the invariant part of the natural history of pre-clinical breast cancer are indeed the same, whereas the increase in the sensitivity reflects the improvement in mammography. Taking the obvious differences between HIP and Nijmegen (one of the two Dutch pilot studies) into account, the model shows that there is a good correspondence between the screening data from these studies. The findings about the duration of pre-clinical disease and the sensitivity of screening can be compared with results from other modelling approaches. Day and colleagues [13] applied this model to data from Utrecht (the other Dutch pilot study). The study reports a good fit of the model (chi-square of 7.2 and 7 degrees of freedom) when assuming a sensitivity of $99 \%$ and a mean duration of 2.8 years. It is not indicated exactly what data from Utrecht were used, but it is clearly a less detailed subset of the data than we used for testing model assumptions. An adapted version of the Day and Walter model was applied to the Nijmegen data [14]. In general, the 


\section{Figure 2}
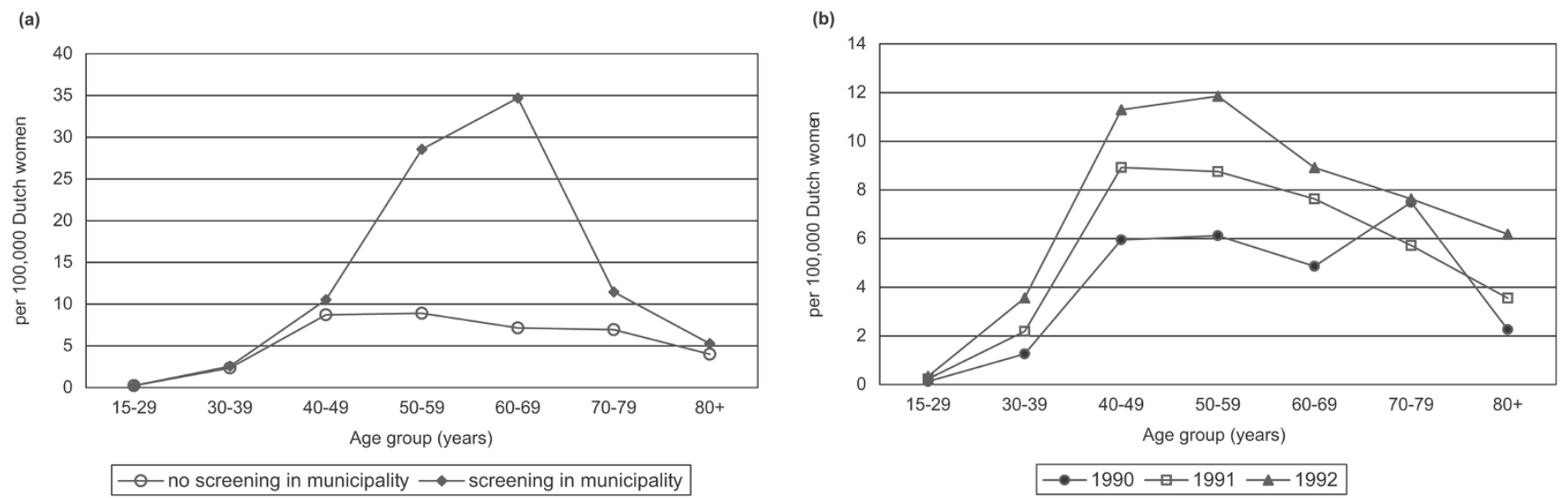

Hospital admissions for non-invasive breast cancer in the early years (1990 to 1992) of screening. (a) Admissions in Dutch municipalities with screening compared to those with no screening. (b) Admissions per calendar year in municipalities without screening.

estimated parameters are comparable to the values found with the MISCAN approach presented here, especially regarding the age-dependency of the estimated duration of the preclinical stage. The reported average duration is somewhat shorter, however, for example, 2.5 years in the 50 to 64 year old age group.

Data on the natural history at older ages have been very limited, but are slowly emerging now that the Dutch programme includes women aged 70 to 74 years [15]. Data on the natural history of DCIS are scarce [16], but parameters concerning the screen-detectable pre-clinical period can be estimated, based on the aforementioned data.

In our first analyses, we have assumed that $10 \%$ of invasive breast cancers are preceded by a screen-detectable DCIS phase and that the chance of progressing to invasive cancer or clinical DCIS is almost $90 \%$ in the long term. Recent data from randomised treatment trials support a high progression rate in the long term [17]. The observed screen data are then consistent/compatible with a mammography sensitivity of $40 \%$ and a mean screen-detectable duration of 5 years.

\section{Results on overdiagnosis}

Figure $3 a, b$ shows the model-estimated changes in breast cancer incidence (by age) in the Netherlands in a programme for women aged 50 to 74 years screened every 2 years (assuming an 80\% attendance rate), compared to no screening. Incidence rises at the starting ages, because all young women have (in principle) never been screened before, which means that cancers are detected that have already progressed over time through the pre-clinical stages. Figure $3 a, b$ also clearly depicts the true extent of overdiagnosis. Because of the earlier detection, cancers that would have surfaced at ages 75 to 85 years have now been detected earlier; clinical incidence at these ages must, therefore, be lower. In Fig. 3a,b, the difference between the left area (extra cancers detected by screening) and the right area (less cancers diagnosed clinically) represents overdiagnosis. We had estimated this to be $3 \%$ of the total incidence, or $8 \%$ of screen-detected cancers. The Dutch data clearly show the decline in incidence at around age 80 years. The higher incidence than expected by the model around ages 55 to 65 years in 2002 (as estimated before the Dutch programme started) confirms the better screening performance in more recent years. It also illustrates the difficulty of estimating overdiagnosis in a situation where a nation-wide screening programme is being implemented.

During the first years of screening, the increase in newly diagnosed cases in the age group invited to screening will not yet be reflected by a decrease in incidence at older ages as these are different cohorts of women. In the later years of screening, the increase in newly diagnosed cases and the decrease in incidence should be at a steady state, although this isn't always the case, because of other changes in the screening programme.

Figure $3 b$ shows that the change in DCIS detection is especially striking, although some lesions would have progressed to an invasive disease (not shown in the figure). The amount of overdiagnosis, the increase in primary surgery/

This article is part of a review series on Overdiagnosis and overtreatment of breast cancer, edited by Nick E Day, Stephen Duffy and Eugenio Paci.

Other articles in the series can be found online at http://breast-cancer-research.com/articles/ review-series.asp?series=BCR_Overdiagnosis 

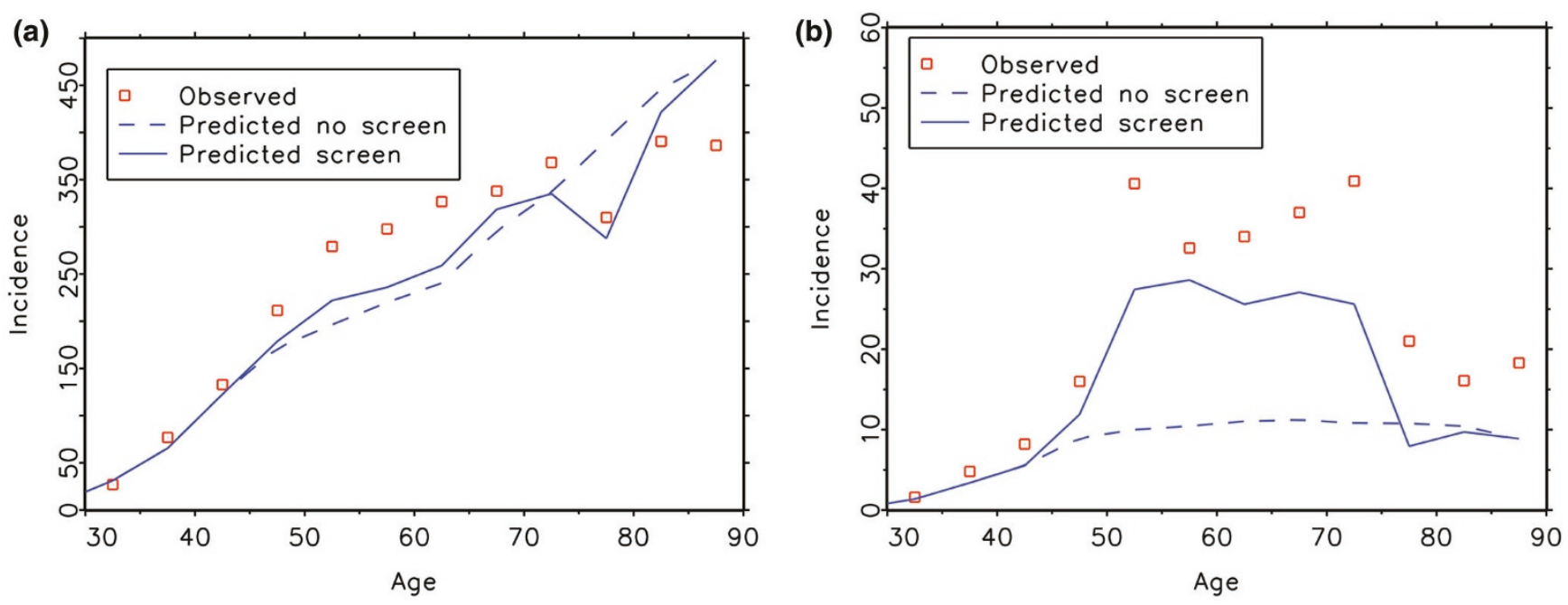

Model estimations of breast cancer incidence. Predicted and observed rates are shown for (a) total incidence and (b) DCIS incidence in the 50 to 74 year old age group with screening every 2 years (assuming 80\% attendance rate) or without screening in the Netherlands in 2002 [8].

radiotherapy, and the longer time frame since diagnosis has to be weighed against the favourable effects of screening: about 750 breast cancer deaths prevented per year (16\%), reduction of treatments for advanced disease and its consequences for quality of life, and 15 life-years gained if dying from breast cancer has been prevented. We consider this to be a very acceptable balance at the population level [18].

\section{Conclusion}

Overdiagnosis is inherent to screening. The crucial issue is the extent to which it happens and what the consequences are for the population involved. This then has to balance with the favourable effects of screening in order to be able to decide on an appropriate screening policy. In breast cancer screening, overdiagnosis is not negligible but is relatively limited. The increases in DCIS are primarily due to mammography screening, but they remain a relatively small proportion of the breast cancer problem. The screen data observed in this study provide workable assumptions on the natural history of DCIS and do not lead to a major difference in conclusions regarding overdiagnosis. More and more women with DCIS are being treated by breast conservation, and in the Netherlands screen-detected DCIS is more often treated by conservation than clinically diagnosed DCIS. Categorisation of DCIS lesions into high risk versus low risk lesions (by screening) is still urgently needed.

\section{Competing interests}

The author(s) declare that they have no competing interests.

\section{References}

1. Nyström L, Andersson I, Bjurstam N, Frisell J, Nordenskjold B, Rutqvist LE: Long-term effects of mammography screening: updated overview of the Swedish randomised trials. Lancet 2002, 359:909-919. Erratum in Lancet 2002, 360:724.
2. Otto SJ, Fracheboud J, Looman CWN, Broeders MJM, Boer R, Hendriks JHCL, Verbeek ALM, de Koning HJ and the National Evaluation Team for Breast Cancer Screening: Initiation of population-based mammography screening in Dutch municipalities and effect on breast-cancer mortality: a systematic review. Lancet 2003, 361:1411-1417.

3. Olsen $\mathrm{AH}$, Nior $\mathrm{SH}$, Vejborg I, Schwartz W, Dalgaard $\mathrm{P}$, Jensen MB, Tange UB, Blichert-Toft M, Rank F, Mouridsen H, Lynge E: Breast cancer mortality in Copenhagen after introduction of mammography screening: cohort study. Br Med J 2005, 330: 220-224.

4. Rijnsburger AJ, van Oortmarssen GJ, Boer R, Draisma G, To T, Miller $A B$, de Koning $\mathrm{HJ}$ : Mammography benefit in the Canadian National Breast Screening Study-2: a model evaluation. Int J Cancer 2004, 110:756-762.

5. de Koning $\mathrm{HJ}$ : Assessment of nationwide cancer-screening programmes - Commentary. Lancet 2000, 355:80-81.

6. van Oortmarssen G: Evaluation of mass screening for cancer: a model-based approach. PhD Thesis. Department of Public Health, Erasmus Universiteit, Rotterdam; 1995.

7. Fracheboud J, Otto S, Dijck JAAM van, Broeders MJM, Verbeek ALM, de Koning HJ, and the National Evaluation Team for Breast Cancer Screening: Decreased rates of advanced breast cancer due to mammography screening in The Netherlands. $\mathrm{Br} J$ Cancer 2004, 91:861-867.

8. Netherlands Cancer Registry: Trends in breast cancer incidence 2002. Utrecht; 2005.

9. Groenewoud JH, Pijnappel RM, van den Akker-van Marle ME Birnie E, Buijs-van der Woude T, Mali WPTM, de Koning HJ, Buskens E: Cost-effectiveness of stereotactic large-core needle biopsy for nonpalpable breast lesions compared to open-breast biopsy. Br J Cancer 2004, 90:383-392.

10. Boer R, Plevritis S, Clarke L: Diversity of model approaches for breast cancer screening: a review of model assumptions by the Cancer Intervention and Surveillance Network (CISNET) Breast Cancer Groups. Stat Meth Med Res 2004, 13:1-14.

11. Day NE, Walter SD: Simplified models of screening for chronic disease: estimation procedures from mass screening programmes. Biometrics 1984, 40:1-14.

12. van Oortmarssen GJ, Boer R, Habbema JD: Modelling issues in cancer screening. Stat Methods Med Res 1995, 4:33-54.

13. Day NE, Walter SD, Tabar L, Fagerberg CJG, Collette HJA: The sensitivity and lead time of breast cancer screening: a comparison of the results of different studies. In Screening for Breast Cancer. Edited by Day NE, Miller AB. Toronto: H Huber Publishers; 1988:105-109. 
14. Verbeek ALM, Straatman H, Hendriks JHCL: Sensitivity of mammography in Nijmegen women under age 50: some trials with the Eddy model. In Screening for Breast Cancer. Edited by Day NE, Miller AB. Toronto: H Huber Publishers; 1988:29-32.

15. Fracheboud J, Groenewoud JH, Boer R, Draisma G, de Bruijn AE, Verbeek ALM, de Koning HJ: The upper age limit of $\mathbf{7 5}$ for breast cancer screening: results from the Dutch nation-wide screening programme. Int $J$ Cancer, in press.

16. Duffy SW, Tabar L, Vitak B, Day NE, Smith RA, Chen HH, Yen MF: The relative contributions of screen-detected in situ and invasive breast carcinomas in reducing mortality from the disease. Eur J Cancer 2003, 39:1755-1760.

17. Julien JP, Bijker N, Fentiman IS, Peterse JL, Delledonne V, Rouanet $\mathrm{P}$, et al.: Radiotherapy in breast-conserving treatment for ductal carcinoma in situ: first results of the EORTC randomised phase III trial 10853. EORTC Breast Cancer Cooperative Group and EORTC Radiotherapy Group. Lancet 2000, 355:528-533.

18. de Haes JC, de Koning HJ, van Oortmarssen GJ, van Agt HM, de Bruyn $A E$, van der Maas PJ: The impact of a breast cancer screening programme on quality-adjusted life-years. Int $J$ Cancer 1991, 49:538-544. 